\title{
A NOTE ON THE LOCATION OF COMPLEX ZEROS OF SOLUTIONS OF LINEAR DIFFERENTIAL EQUATIONS
}

\author{
STEVEN B. BANK
}

1. Introduction. For second-order equations, $w^{\prime \prime}+A(z) w=0$, where $A(z)=a_{m} z^{m}+\cdots$ is a polynomial of degree $m \geq 1$, there is a classical result (due jointly to $E$. Hille, R. Nevanlinna and $H$. Wittich $[10$, p. 282]) which determines the possible location of the zeros of any solution $f \not \equiv 0$. The theorem states that for any $\varepsilon>0$, all but finitely many zeros of $f$ lie in the union (for $j=0,1, \ldots, m+1$ ) of the $\varepsilon$-sectors, $\left|\arg z-\phi_{j}\right|<\varepsilon$, where $\phi_{j}=(2 \pi j-c) /(m+2)$ for any choice of $c=\arg a_{m}$. (The rays $\arg z=\phi_{j}$ are called "critical rays".) In this paper, we determine the situation for higherorder equations.

$$
w^{(n)}+a_{n-1}(z) w^{(n-1)}+\cdots+a_{1}(z) w^{\prime}+a_{0}(z) w=0 \quad(n \geq 2)
$$

where the $a_{j}(z)$ are polynomials. As shown in Theorem 1 below ( $\left.\S 3\right)$, an interesting feature of the higher-order case is that the Hille-Nevanlinna-Wittich property (i.e., the existence of finitely many critical rays around which the zeros of any solution $f \not \equiv 0$ must be concentrated) need not hold when $n>2$. There are equations (e.g. see $\S 4$ below) which have the property that for any ray, and any $\varepsilon$-sector around it, some solution $f \not \equiv 0$ has infinitely many zeros in the $\varepsilon$-sector. In Theorem 1 , we show that in general either this latter property or the Hille-Nevanlinna-Wittich property holds for a given equation (1), and one can easily determine from the equation which of the two holds. In $\S 7$, we consider the problem of explicitly determining the critical rays for those equations (1) possessing the Hille-Nevanlinna-Wittich property.

The key tools in the proof of Theorem 1 are asymptotic existence theorems which were proved in [4] and [6] using the Strodt theory [8, 9]. (Details of the proof will appear elsewhere.)

2. Preliminaries. Given an equation (1) where the $a_{j}(z)$ are any rational functions, we first rewrite the equation (1) in terms of the operator $\theta$ defined by $\theta w=z w^{\prime}$. (It is easy to prove by induction that for each $m=1,2, \ldots$,

$$
w^{(m)}=z^{-m}\left(\sum_{j=1}^{m} b_{j m} \theta^{j} w\right),
$$

Received by the editors June 24, 1987.

1980 Mathematics Subject Classification (1985 Revision). Primary 34A20, 34C10.

Research supported in part by the National Science Foundation (DMS-8420561). 
where $\theta^{j}$ is the $j$ th iterate of the operator $\theta$, and where the $b_{j m}$ are integers with $b_{m m}=1$.) When written in terms of $\theta$, let (1) have the form

$$
\sum_{j=0}^{n} B_{j}(z) \theta^{j} w=0 .
$$

(Of course, the $B_{j}(z)$ are also rational functions.) By dividing the equation (3) through by the highest power of $z$ which occurs in all Laurent expansions of the $B_{j}(z)$ around $z=\infty$, we may assume that for each $j$ we have $B_{j}=O(1)$ as $z \rightarrow \infty$, and there exists an integer $p \geq 0$ such that $B_{j}=o(1)$ as $z \rightarrow \infty$ for $j>p$, while $B_{p}$ has a finite nonzero limit (denoted $B_{p}(\infty)$ ) at $\infty$. By multiplying the equation through by a constant, we may also assume that $B_{q}(\infty)=1$ where $q=\min \left\{j: B_{j}(\infty) \neq 0\right\}$. The integer $p$ is called the critical degree of the equation (1). The equation

$$
F^{*}(\alpha)=\sum_{j=0}^{n} B_{j}(\infty) \alpha^{j}=0
$$

is called the critical equation of (1). (Clearly, $F^{*}(\alpha)$ is a polynomial in $\alpha$, of degree $p$, having constant coefficients.) We define a sequence of integers $p=t(0)<t(1)<\cdots<t(\sigma)=n$ as follows: $t(0)=p$, and if $t(j)$ has been defined and is less than $n$, let $t(j+1)$ be the largest integer $k$ such that $t(j)<k \leq n$ and such that $B_{i}=O\left(B_{k}\right)$ as $z \rightarrow \infty$ for all $i$ satisfying $t(j)<i \leq n$. The polynomial in $v$ defined by

$$
G(v)=\sum_{j=0}^{\sigma} z^{t(j)} B_{t(j)}(z) v^{t(j)-p}
$$

is called the factorization polynomial for (1). Clearly, $G(v)$ is a polynomial of degree $n-p$ in $v$, whose coefficients are rational functions of $z$. (We observe also that $G(0) \not \equiv 0$.) We will say that "the roots of $G(v)$ are asymptotically distinct" if the $n-p$ possible expansions around $z=\infty$ of the algebraic function defined by $G(v)=0$ all have different first terms. It is easy to see that each first term is a function of the form $c z^{\alpha}$ where $\alpha$ is a rational number exceeding -1 , and $c$ is a nonzero complex constant. In the terminology of [4], these functions $c z^{\alpha}$ are the critical monomials of $G(v)$, and can be produced by the Newton polygon method described in [7, p. 105].

3. THEOREM 1. Given the equation (1) where the $a_{j}(z)$ are polynomials, then:

(A) If two distinct roots of the critical equation for (1) have the same real part, then (1) has the following property: For any $\varepsilon>0$ and any value of $\phi$ in $(-\pi, \pi]$, there exists a solution $f \not \equiv 0$ of (1) which has infinitely many zeros in the $\varepsilon$-sector $|\arg z-\phi|<\varepsilon$.

(B) Assume that no two distinct roots of the critical equation for (1) have the same real part, and assume further that the roots of the factorization polynomial for (1) are asymptotically distinct. Then there exist a positive integer $m$ and finitely many real numbers $\sigma_{1}, \ldots, \sigma_{m}$ in $(-\pi, \pi]$ such that for any 
$\varepsilon>0$ and any solution $f \not \equiv 0$ of (1), all but finitely many zeros of $f$ lie in the union for $j=1, \ldots, m$ of the $\varepsilon$-sectors $\left|\arg z-\sigma_{j}\right|<\varepsilon$.

4. Example. For $w^{(n)}+z^{2} w^{\prime \prime}+z w^{\prime}+w=0$ where $n>2$, the critical equation is $\alpha^{2}+1=0$, and so (A) holds.

5. Notation. We will use the notation $F(a, b)$ introduced in $[8, \S 94]$ to denote the neighborhood system of sectorial regions which are approximately of the form $a<\arg \left(z-z_{0}\right)<b$, where $\arg z_{0}=(a+b) / 2$. We will also use the asymptotic relations $f_{1} \sim f_{2}, f_{1} \ll f_{2}$, and $f_{1} \approx f_{2}$ over $F(a, b)$ developed in $[8, \S 13]$, which can be thought of respectively as $f_{1} / f_{2} \rightarrow 1, f_{1} / f_{2} \rightarrow 0$, and $f_{1} / f_{2} \rightarrow c$ for some nonzero constant $c$, as $z \rightarrow \infty$ over $F(a, b)$.

6. Proof of Theorem 1. Part (A). Assume that the critical equation for (1) possesses distinct roots $\alpha$ and $\beta$ with $\alpha-\beta=s i$, where $s>0$. By $[2, \S 7]$, there exist finitely many points $\phi_{1}<\phi_{2}<\cdots<\phi_{q}$ in $(-\pi, \pi)$ such that in each of $F\left(-\pi, \phi_{1}\right), F\left(\phi_{1}, \phi_{2}\right), \ldots, F\left(\phi_{q}, \pi\right)$ separately, the equation (1) possesses solutions $g_{1}$ and $g_{2}$ with $g_{1} \sim z^{\alpha}$ and $g_{2} \sim z^{\beta}$. Let $\phi$ be any number in $(-\pi, \pi)$ which is distinct from $\phi_{1}, \ldots, \phi_{q}$, and let $\varepsilon>0$ be arbitrary. We can assume $\varepsilon$ is so small that $g_{1}$ and $g_{2}$ are analytic in some element of $F(\phi-\varepsilon, \phi+\varepsilon)$, and satisfy $g_{1} \sim z^{\alpha}$ and $g_{2} \sim z^{\beta}$. Using [5, Lemma D, p. 127] and Rouché's theorem, we produce infinitely many zeros of $\left(g_{1} / g_{2}\right)-e^{-s \phi}$ lying in $|\arg z-\phi|<\varepsilon$. Thus this $\varepsilon$-sector contains infinitely many zeros of the solution $g_{1}-e^{-s \phi} g_{2}$. (It follows easily that Part (A) then also holds when $\phi$ is equal to one of the points $\phi_{1}, \ldots, \phi_{q}, \pi$.)

Assume the hypothesis of Part (B) and let $p$ be the critical degree of (1) and let $q$ be as in $\S 2$. Let the distinct nonzero roots of the critical equation of (1) be $\alpha_{1}, \ldots, \alpha_{r}$, with $\alpha_{j}$ having multiplicity $m_{j}$. (Thus $q+\sum m_{j}=p$.) Define $M_{1}, \ldots, M_{p}$ as follows: $M_{j}=(\log z)^{j-1}$ if $1 \leq j \leq q ; M_{q+j}=z^{\alpha_{1}}(\log z)^{j-1}$ if $1 \leq j \leq m_{1}$, and in general,

$$
M_{q+m_{1}+\cdots+m_{k}+j}=z^{\alpha_{k+1}}(\log z)^{j-1}
$$

for $1 \leq k<r$ and $1 \leq j \leq m_{k+1}$. Let $N_{1}, \ldots, N_{n-p}$ be the distinct critical monomials (see §2) of the factorization polynomial for (1). It is proved in [4, §3] that there are finitely many points $-\pi=r_{0}<r_{1}<\cdots<r_{t}<r_{t+1}=\pi$ such that in each $F\left(r_{j}, r_{j+1}\right)$, the equation (1) possesses a fundamental set of solutions $\left\{g_{1}, \ldots, g_{p}, h_{1}, \ldots, h_{n-p}\right\}$, where $g_{j} \sim M_{j}$ for $1 \leq j \leq p$, and each $h_{k}$ is of the form $\exp \int V_{k}$, where $V_{k} \sim N_{k}$ for $1 \leq k \leq n-p$. In view of the hypothesis, it can be shown (using $[1, \S 10]$, and a variant of $[8, \S 41]$ ) that in $F\left(r_{j}, r_{j+1}\right)$, the ratio of any two distinct solutions in the fundamental set either tends to zero or to $\infty$. Thus if $f \not \equiv 0$ is any solution of (1), then since $f$ is a nontrivial linear combination of $g_{1}, \ldots, g_{p}, h_{1}, \ldots, h_{n-p}$, it easily follows that $f$ has no zeros on some element of $F\left(r_{j}, r_{j+1}\right)$, and the proof of Part (B) follows when we take $\left\{\sigma_{1}, \ldots, \sigma_{m}\right\}$ to be $\left\{r_{1}, \ldots, r_{t+1}\right\}$.

7. Remark. For certain classes of equations (1) whose coefficients are rational functions, it can be shown that the critical rays can be explicitly calculated. For example, this is the case for those equations which satisfy the following two conditions: (i) The roots of the factorization polynomial are 
asymptotically distinct; (ii) The roots of the critical equation are all simple, and no two distinct roots have the same real part.

\section{REFERENCES}

1. S. Bank, On the asymptotic behavior of solutions near an irregular singularity, Proc. Amer. Math. Soc. 18 (1967), 15-21.

2. __, An asymptotic analog of the Fuchs regularity theorem, J. Math. Anal. Appl. 16 (1966), 138-151.

3. _ On solutions having large rate of growth for nonlinear differential equations in the complex domain, J. Math. Anal. Appl. 22 (1968), 129-143.

4. - On the structure of a fundamental set of solutions near an irregular singularity, Funkcial. Ekvac. 11 (1968), 87-100.

5. $\ldots$, On the location of complex zeros of solutions of periodic differential equations, Appl. Anal. 22 (1986), 111-132.

6. E. W. Chamberlain, Families of principal solutions of ordinary differential equations, Trans. Amer. Math. Soc. 107 (1963), 261-272.

7. E. Hille, Analytic function theory, Volume II, Chelsea, New York, 1973.

8. W. Strodt, Contributions to the asymptotic theory of ordinary differential equations in the complex domain, Mem. Amer. Math. Soc., No. 13, 1954.

9. __ Principal solutions of ordinary differential equations in the complex domain, Mem. Amer. Math. Soc., No. 26, 1957.

10. H. Wittich, Eindeutige Lösungen der Differentialgleichung $w^{\prime}=R(z, w)$, Math. Z. 74 (1960), 278-288.

Department of Mathematics, University of Illinois, Urbana, Illinois 61801 